\title{
EDITORIAL
}

\section{Advancing the Visibility, Impact, and Quality Metrics of Value in Health Regional Issues}

We are happy to present the fifth volume of Value in Health Regional Issues (ViHRI), focusing on Central and Eastern Europe, Western Asia, and Africa (CEEWAA). The first issue of the journal focusing on articles from Asia was published in May 2012, whereas the first issue devoted to the CEEWAA countries was published in September 2013. Since then, the journal has made an impressive progress. At the end of 2016, ViHRI was accepted for indexing in MEDLINE and PubMed. All articles published since May 2016 are already indexed and articles published in previous volumes are expected to be indexed soon. The journal's indexing allows for content published to be more accessible, more searchable, and more citable to researchers around the world. This is a great achievement for the journal and the International Society for Pharmacoeconomics and Outcomes Research (ISPOR). Academic scholars aim at publishing their research in journals that are indexed in large databases (e.g., MEDLINE) and are of high quality, usually measured by the journal's impact factor and its rank in a specific discipline, such as economics or health policy and services. The most common and largest database that systematically tracks citation data of journals and computes measures of quality is the Clarivate Analytics Journal Citation Reports (JCR). In 2016, JCR covered 11,365 journals from 234 disciplines [1]. For example, the 2016 2-year impact factor of Value in Health (ViH), the official journal of the ISPOR, was 4.235 (5-year impact factor of 4.491). The journal is ranked seventh among 347 journals in economics, third among 77 journals in health policy and services, and ninth among 90 journals in health care sciences and services.

The SCImago Journal \& Country Rank (SJR) is a publicly available portal that includes the journals and country scientific indicators developed from the information contained in the

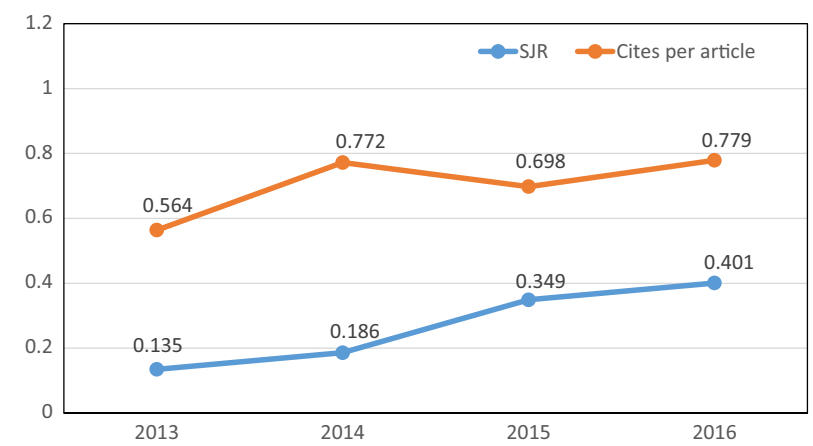

Fig. 1 - SJR scores and cites per article (Value in Health Regional Issues 2013-2016). SJR, SCImago Journal \& Country Rank.
Scopus database (Elsevier B.V.). SJR is a measure of the scientific influence of journals that accounts for both the number of citations received by a journal and the importance or prestige of the journals from which such citations come. Citation data are drawn from more than 21,500 titles from more than 5,000 international publishers. Journals are grouped by subject area (27 major thematic areas), subject category (313 specific subject categories), or country [2]. The correlation between the SJR and the JCR is very high and was approximately 0.99 in a sample of 64 economics journals [3].

Since the first publication of ViHRI in 2012, the journal has not yet been indexed in the JCR. An analysis of SJR scores and cites per article from 2013 through 2016 suggests that the quality and influence of the journal are constantly improving (Fig. 1). The SJR score has improved from 0.135 in 2013 to 0.401 in 2016. According to this score, ViHRI is in the second quartile of journals in each of the following disciplines: economics, econometrics, and finance; health policy; and pharmacology, toxicology, and pharmaceutics [4].

We also compared the recent SJR scores of ViHRI with those obtained for $\mathrm{ViH}$ for the first 4 years (1999-2003) from its first publication. As presented in Figure 2, where "year 1" indicates the year 1999 for ViH and the year 2013 for ViHRI, the scores for both the ISPOR journals are comparable.

Furthermore, CiteScore is another measure of the impact of articles published in a journal. The score measures the average citations received per document published in the journal. CiteScore values are based on citation counts in a given year (e.g., 2016) to documents published in the previous 3 calendar years (e.g., 2013-2015) divided by the number of documents in these previous 3 years. The CiteScore value for ViHRI for 2016 was 0.71 (135 citations in 2016 for 191 documents published in 2013-2015), an increase from 0.57 in 2015.

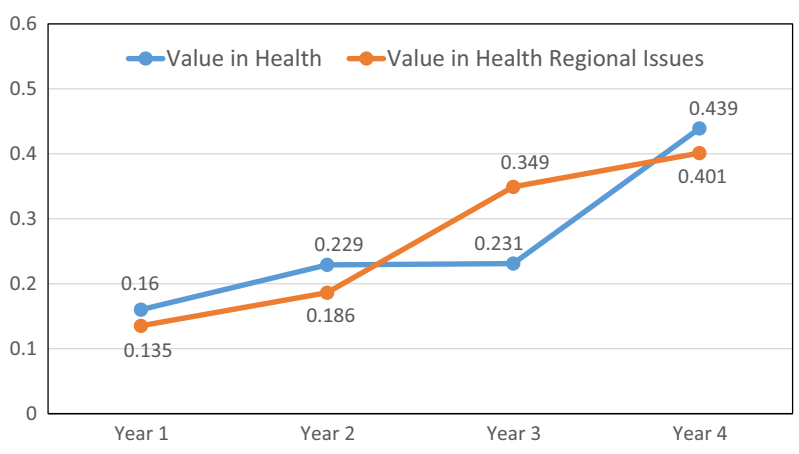

Fig. 2 - SJR scores for Value in Health and Value in Health Regional Issues. SJR, SCImago Journal \& Country Rank. 
It is noteworthy that articles published in the journal are made available online via the ScienceDirect platform. Elsevier tracks the number of full-text downloads received by each journal, providing a good indication of the trend in article usage. The number of downloads has increased substantially in recent years from approximately 10,500 in 2013 to almost 30,000 in 2016. The data gathered for 2017 suggest that the number of downloads will further increase.

This progress could not have been achieved without the substantial support from the ISPOR staff as well as Elsevier, the publisher of the journal. We are confident that the quality of manuscripts submitted and the journal's quality metrics will further increase in the coming years. We are still making efforts to speed up the review process and the time from manuscript acceptance to online publications. For this purpose, we are now using a more user-friendly editorial and submission system (ScholarOne). We encourage our readers to submit their highquality manuscripts for publication in our journal.

As part of the strategic planning for ViHRI, we publish in this volume, for the first time, a theme section on drug policies in Central and Eastern Europe (CEE). The project was initiated and conducted by the ISPOR CEE Publication Network Working Group. In this volume, we present the first part of the theme section including reports from seven countries. Additional articles from other CEE countries will be included in the next volume. We thank the project's committee and guest editors of the section, Karina Janhz-Różyk, Paweł Kawalec, and Monika SzkulteckaDebek, for their major contributions. Another collaboration among researchers from various African countries discusses the health economic challenges, lessons learned, and policy recommendation of Ebola infection in West African countries [5]. Other interesting and topical issues from different parts of CEEWAA also feature in this volume. We encourage scholars in CEEWAA countries to collaborate and submit studies and policy perspectives pertaining to several countries and health care systems in their region. A good example is a study reporting on the cost burden of severe community-acquired rotavirus gastroenteritis requiring hospitalization in the Czech Republic, Slovakia, Poland, and Hungary published in our previous volume [6]. Aleš Tichopád, the lead author of this article, will be presented with the ViHRI Excellent Article Award for his contribution.

Recently, we have issued a general call for articles for research that explores the application of health technology assessments, health policy analysis, and drug policies for biosimilars in health care decision making for patient populations. Nevertheless, we accept contributions from all areas of pharmacoeconomics and outcomes research.

Finally, we thank Malgorzata (Gosia) Juszczak-Punwaney, Steve Priori, and Lyn Beamesderfer from ISPOR for their editorial support and Terry Materese from Elsevier, the journal's publisher, for providing the journal's metrics. We also thank
Mohamed Izham Mohamed Ibrahim, the previous co-editor of ViHRI for the CEEWAA region, for his excellent contribution to the journal and to ISPOR.

We hope that you enjoy reading the articles in this volume.

Dan Greenberg, PhD

Department of Health Systems Management Ben-Gurion University of the Negev Be'er Sheva, Israel

Imre Boncz, MD, MSc, PhD, Habil Faculty of Health Sciences Institute for Health Insurance

University of Pécs Pécs, Hungary

Ahmed Awaisu, PhD, BPharm College of Pharmacy

Qatar University Doha, Qatar

2212-1099/\$36.00 - see front matter (c) 2017 Published by Elsevier Inc. on behalf of International Society for Pharmacoeconomics and Outcomes Research (ISPOR). http://dx.doi.org/10.1016/j.vhri.2017.08.007

R E F E R E N C E S

[1] Thomson Reuters. 2016. Available from: http://scientific thomsonreuters.com/imgblast/JCRFullCovlist-2016.pdf. [Accessed August 20, 2017]. (Journal Citation Reports).

[2] SCImago. SJR-SCImago Journal \& Country Rank. Available from: http:// www.scimagojr.com. [Accessed August 21, 2017].

[3] Azar O. The impact factor and ranking of the Journal of SocioEconomics. J Socio Econ 2013;42:142-3.

[4] Scimago Journal \& Country Rank. Available from: http://www.scimagojr. com/journalsearch.php?q=21100218529\&tip=sid\&clean=0. [Accessed August 20, 2017].

[5] Elmahdawy M, Elsisi G, Carapinha J, et al. Ebola virus epidemic in West Africa: global health economic challenges, lessons learned, and policy recommendations. Value Health Reg Issues 2017;13:67-70.

[6] Tichopád A, Müllerová J, Teresa Jackowska T, et al. Cost burden of severe community-acquired rotavirus gastroenteritis requiring hospitalization in the Czech Republic, Slovakia, Poland, and Hungary: a retrospective patient chart review. Value Health Reg Issues 2016;10:53-60. 